\title{
Towards an Emergency Nursing Specialization in Sudan
}

\author{
Dr. Hassanat E. Mustafa and Dr. Badria M. Elfaki \\ Faculty of Nursing, Al Neelain University, Khartoum, Sudan, Department of Nursing Practice at Nursing \\ collage in Um Al Qura University, Makkah. , Kingdom of Saudi Arabia
}

\begin{abstract}
:
Background: An emergency nursing is the nursing specialty that encompasses caring for patients of all age groups and conditions, from delivering babies and resuscitating trauma arrests to administering medication. The pace of emergency nursing also varies from slow to hyper drive, and it can change within minutes.

Objective of this article was to assess the roles, responsibilities and training of nurses at emergency departments at three public hospitals at Khartoum state in Sudan.

Method: Descriptive review study design was carried out at Khartoum, Khartoum north and Omdurman hospitals emergency departments. Interview was carried out in 2010 for all nurses who worked at these departments.

Results: All nurses worked; at these emergency departments had a bachelor degree, No nursing speciality. The nurses received patients. Check vital signs applied intravenous cannula and dressing. Also they applied ECG for the cardiac patients. Also nurses gave intravenous fluids, medication in addition to monitoring the patients. $90 \%$ of the staff hadn't received training; but only $10 \%$ received training about basic and advanced life support. The triage system in these hospitals performed just in sorting room with one nurse staff to sort all patients who came to an emergency department.

Conclusion: There was no emergency nursing and no speciality in the Sudan before; and no triage system. The plan for building emergency departments in specific design started in 2005 at each hospitals then, selection and training for the staff for various services.

Keywords: Emergency, Speciality, Sudan, Nursing.
\end{abstract}

\section{Introduction}

An emergency nursing is a nursing specialisation in which the nurses care for patients in a critical phase of the illness or injury. The emergency nurse's skill in caring for a patient before a diagnosis has been made or the cause of the complaint known .Nurses in this type of situation must be highly competent, skilled and confident in order to cope with a wide variety of emergency situations, many of them may be a lifethreatening [1]. Currently in Sudan the Federal Ministry of Health is focusing on providing quality health care to all citizens [2]. Its desire is to meet the expectation of the Sudan people and promote their health and wellbeing [3]. Also and emergency departments need triaging system to facilitate care of the patient and to prevent any delay of management .Standardized international triage system is organized with high training nurses staff they received patients, assessed and checked their vital signs then transfer each one according the degree of illness organized from hot cases to cold one using different colour chart to facilitate sorting patients from high to low emergency e.g. red colour chart for high serious cases and no chance for delay.

The article aim was to assess the roles, responsibilities and training of nurses at emergency departments in Sudan and comparing with the emergency services in British, UK and Ireland in order to support the emergency nursing in the Sudan, gain their co-operation and to propose steps to develop a nursing specialisation in emergency nursing since 2010.

\section{Methods}

Review services which were performed by nurses at various emergency departments and critical care units; at three public teaching hospitals in Khartoum state. The emergency departments were divided into different rooms A, B, C, T and triage area. Interviews were carried out for total coverage for 50 nurses, who work in the morning shift; to determine their' roles for patients at each room. As well as a key interview was carried out with each head nurse staff of these departments and administrators.

The interview questionnaire rolled out the role of the nurses at emergency departments; numbers of the patients regard number of staff and sort of training for nursing staff and check for triage system. The interview carried for three sequences days. The collected data was summarized manually and the finding was presented at pre workshop of a conference which carried at Continuing Professional Development (CPD) centre at Khartoum state followed by a comparative presentation about emergency departments at Khartoum, UK and Ireland. 


\section{Results}

In rooms A, B or ICU; the nurse's tasks are critical to the patients, after connecting a patient to a monitor takes a blood sample and sent the sample to the lab for requested investigations then perform an electrocardiogram (ECG) trace while continually keeping an eye on the vital signs. As a patient's stay continues the nurse must take daily care for all needs of the patient and begin to teach the patient and relatives how to do the same when the patient is discharged.

Nurses in room C: when they are dealing with patients who are having an asthma attack have several tasks to perform after receiving the patients, all of which must be done quickly and efficiently. The nurse needs to insert an IV cannula, take a blood sample, administer oxygen and other requested medicine and carry out any other nursing procedures are required.

A patient in the $\mathrm{T}$ room may need a wound dressed or sutured, tasks performed by a nurse. In the sorting room the nurse received the patients, registers their personal data and sorts them according to the type and seriousness of their complaint then sent the patient to the relevant department of the hospital.

\section{The structure of the emergency departments:}

\section{Discussions}

In Sudan a new emergency department structures have been established in the teaching hospitals 'Khartoum, Khartoum North and Omdurman' [4]. The main change has been in implementing assorting system and available resources are widely utilised and the burden on other parts of the health service minimised. Compare there are a similarity in the structure between Sudan an UK and Ireland emergency departments, but their Emergency Department (ED) are wider and there are some differences in performing some services such as triaging patients and modern methods for transferring patients via ambulances. A key difference with specially trained nurses; the triage system specify the patient according to their problem, acute level or emergency; using deferent card with specific colour i.e. the red colour chart for severe cases, yellow for low severity as well as green for cold one, then each case received at specific area and received specific service according to his or her problem. The purpose of triage in the Emergency Department (ED) is to prioritize incoming patients and to identify those who cannot wait to be seen [6].

The triage nurse performs a brief, focused assessment and assigns the patient a triage acuity level, which is a proxy measure of how long an individual patient can safely wait for a medical screening examination and treatment. In 2008 there were 123.8 million visits to U.S. emergency departments; of those visits, only $18 \%$ of patients were seen within 15 minutes, leaving the majority of patients waiting in the waiting room. Historically, Emergency Departments (EDs) in the United States did not use standardized triage acuity rating systems. Since 2000, there has been a trend toward standardization of triage acuity scales that have five levels (e.g., 1- resuscitation, 2- emergent, 3- urgent, 4- less urgent, 5- non-urgent [6].

\section{Nurse's staff}

Role of the nurse at an emergency department at Khartoum teaching hospital provides a variety of roles in the emergency department depending on which section or area in which they are stationed. The nurses work as the bees the number of patients are more than nurse capacity. Also the nurses complain for low salary and over load of the work due to the shortage of staff.

As far as emergency nursing is concerned, there is not yet a specialisation in emergency nursing in these hospitals. Also there is shortage in training, but there are a number of courses available for nurses to improve their emergency nursing skills such as Basic and Advance life Support (BLS and ALS) are administered by the Continuing Professional Development (CPD) centre, continuing medical education and professional developing programs in Sudan. Unfortunately less than $1 \%$ received these courses. This was widely disagreed with nurses' staff at UK and Ireland. All things are available for care, number for nurses' staff combatable to the number of patients and all staff training for emergency nursing and have licences and specialization.

Emergency nurses frequently contact patients in the emergency department before the patients see physicians. In this situation, the nurse must be skilled at rapid, accurate physical examination, early recognition of life-threatening illness or injury, the use of advanced monitoring and treatment equipment, and in some cases, the ordering of testing and medication according to "advance treatment guidelines" or "standing orders" set out by the hospital's emergency physician staff. A specialist nurse who will independently assess, diagnose, investigate, and treat a wide range of common accidents and injuries working autonomously without reference to medical staff $[9,10]$.

Due to unplanned nature of patient attendance, the department must provide initial treatment for a broad spectrum of illnesses and injuries, some of which may be life-threatening and require immediate attention. In some countries, emergency departments have become important entry points for those without other means of access to medical care. The emergency departments of most hospitals operate 24 hours a day, although staffing levels may be varied in an attempt to reflect patient volume [8]. 
The education programs have been approved in an attempt to ensure excellent standards of practice are achieved and to improve the level of health services provided [5]. Through ensuring that the medical practitioners and health care personal are continuously updated in relation to their perspective fields; ascertaining that their performance is of the highest levels; verifying that they maintain that level of performance. Also a nurse may be required to accompany the patient to another department. All of these requirements are over and above the normal nursing procedures with which a nurse must be familiar on a given ward such as giving regular medicines, helping with personal hygiene and monitoring vital signs. Intrust of emergency nursing specialty that encompasses caring for patients of all age groups and conditions, the pace of emergency nursing also varies from slow to hyper drive, and it can change within minutes [7]. Due to complexity of emergency nursing the characteristics of nursing staff that will serve well as an emergency nurse such as ability to shift gears and accelerate the pace as needed, good observation, assessment, and prioritization skills, multi-tasking ability, good interpersonal and customer service skills good personal coping skills, assertive patient advocate good sense of humour and ability to think fast [7].

\section{Challenges To Nursing Practice In Sudan}

Perhaps partly because there are so many demands on nurses as described above, there is generally a low morale in the profession in Sudan. Other challenges, all of which may be inter-related, are low salaries, heavy workload, high turnover and the attraction of working abroad.

\section{The Way Forward}

It is our belief that one means of addressing these challenges and improving emergency nursing care is to promote emergency nursing as a specialisation. It is difficult to see how developing nurses who are highly trained in those tasks unique to the emergency department to improve the whole emergency room operation. Of course, commitment to such a training programme will mean very careful monitoring and ongoing supervision of the emergency nurses. They would need to have a continuous professional development (CPD) scheme that allowed them to take advantage of best practice advice from other parts of the world and develop it into protocols and guidelines right for the Sudanese setting one element of the emergency room of special importance in this scheme is the triage system. In future, specialist emergency nurses can and shall be part of the ambulance team sent to emergency situations outside the hospital.

\section{VII.Conclusion}

As far as emergency nursing is concerned, there is not yet a specialisation in Sudan. There are similarity between emergency nursing and emergency department between us and UK and Ireland, but we need to collaborate together to face these challenges and to improve our performance.

Prof: Mustafa Numiri for guidance and reviewing

\section{Acknowledgment}

Prof: Andy Mcconkey Elneelain University: for helping and reviewing

Sister Alawia Ahamed Ministry of health (MOH)/ nursing department) for her help

Prof. Grace Lindy Umm Al Qura University for reviewing.

\section{References}

[1]. Wikipedia(2010http://en.wikipedia.org/wiki/Emergency_nursing)

[2]. http://www.linkedin.com/pub/faiz-mohammed-ahmed/28/256/399

[3]. http://www.fmoh.gov.sd/English/index.php

[4]. Sara Am abdElaal, Yousif A./Sudanese (2005) waiting time at emergency departments/Journal of Public Health website:http://www.sjph.net.sd/files/v1i2p122-126.pdf

[5]. Continuous professional development (CPD) : 2010 training for professionals http://www.cpd.gov.sd/

[6]. Agency for healthcare Research and Quality (2014) Advancing Excellence in Health Care Emergency Severity Index (ESI): A Triage Tool for Emergency Department Emergency medical services in the United Kingdom.

[7]. [7] 2015 Chicago Tribune story provides an overview of emergency nursing as a career.

[8]. https://www.ena.org/membership/Pages/WhyEmergencyNursing.aspx

[9]. [8] Wikipedia, the free encyclopedia emergency department

[10]. https://en.wikipedia.org/wiki/Emergency_department.

[11]. Wikipedia, (2015 Emergency nursing https://en.wikipedia.org/wiki/Emergency_nursing.

[12]. Anne Manton Emergency nurses are elegant generalists...they must know a great deal about virtually all aspects of nursing. http://www.nsna.org/Portals/0/Skins/NSNA/pdf/Imprint_Jan04_Manton.pdf

[13]. Matthew D. McHugh2011Hospital Nurse Staffing and Public Health Emergency Preparedness: Implications for PolicyPublic Health Nurs. 2010 Sep; 27(5): 442-449.http://www.ncbi.nlm.nih.gov/pmc/articles/PMC2998349/ 


\section{Annexes:}

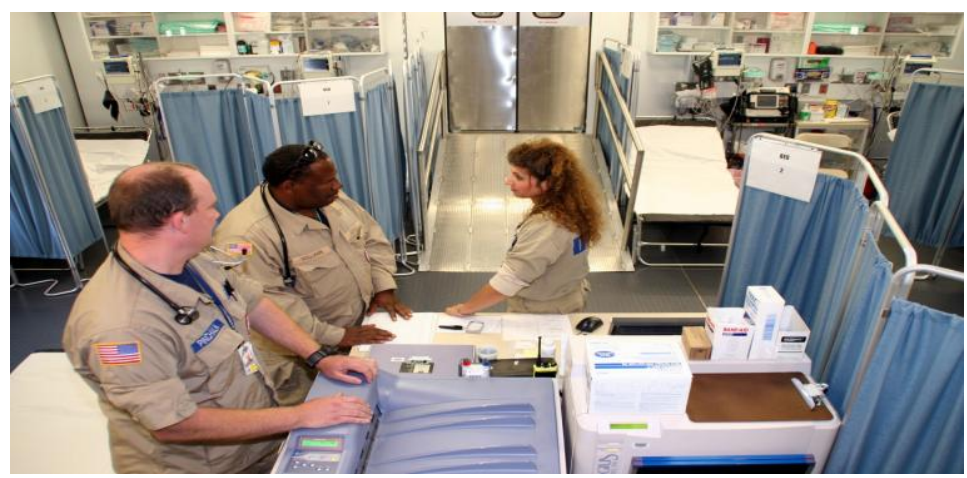

Hospital restructuring policies and an impending nursing workforce shortage have threatened the nation's emergency preparedness. Current emergency response plans rely on sources of nurses that are limited and overestimated. A national investment in nursing education and workforce infrastructure, as well as incentives for hospitals to efficiently maximize nurse staffing, are needed to ensure emergency preparedness in the United States. This review highlights the challenges of maintaining hospital nursing surge capacity and policy implications of a nursing shortage [11].

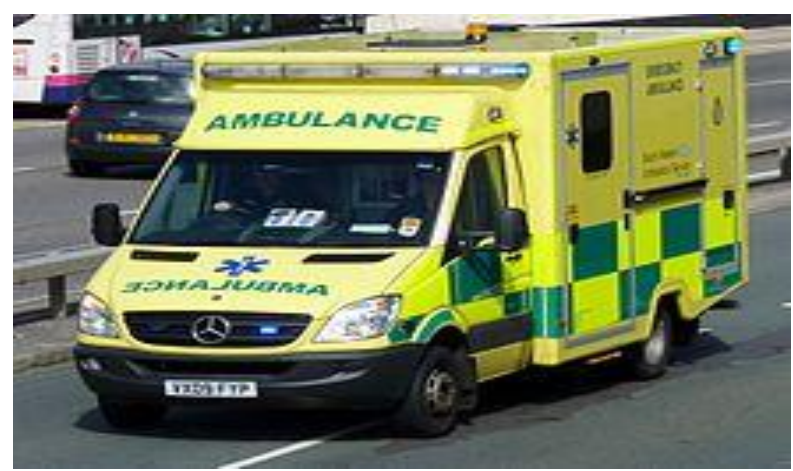

A Mercedes Sprinter ambulance of the South Western Ambulance Service responds to an emergency call

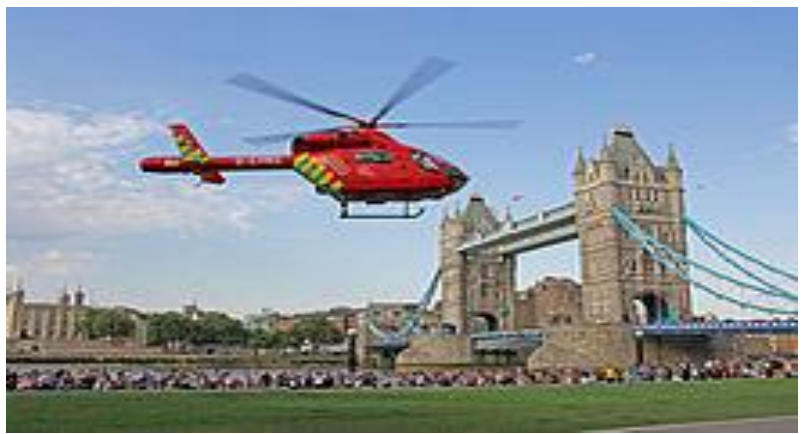

The London Air Ambulance in action

Emergency medical services in the United Kingdom provide emergency care to people with acute illness or injury and are predominantly provided free at the point of use by the four National Health Services of England, Scotland, Wales, and Northern Ireland. Emergency care including ambulance and emergency department treatment is free to everyone, regardless of immigration or visitor status. 